PROCEEDINGS OF THE

AMERICAN MATHEMATICAL SOCIETY

Volume 133, Number 9, Pages 2625-2635

S 0002-9939(05)08068-8

Article electronically published on April 19, 2005

\title{
DIRICHLET BOUNDARY CONDITIONS FOR ELLIPTIC OPERATORS WITH UNBOUNDED DRIFT
}

\author{
A. LUNARDI, G. METAFUNE, AND D. PALLARA
}

(Communicated by David S. Tartakoff)

\begin{abstract}
We study the realisation $A$ of the operator $\mathcal{A}=\Delta-\langle D \Phi, D \cdot\rangle$ in $L^{2}(\Omega, \mu)$ with Dirichlet boundary condition, where $\Omega$ is a possibly unbounded open set in $\mathbb{R}^{N}, \Phi$ is a semi-convex function and the measure $d \mu(x)=$ $\exp (-\Phi(x)) d x$ lets $\mathcal{A}$ be formally self-adjoint. The main result is that $A$ : $D(A)=\left\{u \in H^{2}(\Omega, \mu):\langle D \Phi, D u\rangle \in L^{2}(\Omega, \mu), u=0\right.$ at $\left.\partial \Omega\right\}$ is a dissipative self-adjoint operator in $L^{2}(\Omega, \mu)$.
\end{abstract}

\section{INTRODUCTION}

Second-order elliptic operators with unbounded coefficients in $\mathbb{R}^{N}$ or in unbounded subsets of $\mathbb{R}^{N}$ have been the object of several recent papers; see e.g. 2, 3, 8, 1, 9]. Since the very first studies it was apparent that operators of the type $\mathcal{A} u=\operatorname{Tr} Q(x) D^{2} u(x)+\langle F(x) D u(x)\rangle$, without potential terms, are not well settled in $L^{p}$ spaces with respect to the Lebesgue measure, unless the matrix $Q$ and the vector $F$ satisfy very severe restrictions, such as global Lipschitz continuity (see [9, 7]). It is much more natural and fruitful to work in suitably weighted $L^{p}$ spaces; see [3, 8. This is what we do in this paper. We consider the operator $\mathcal{A}$ defined by

$$
\mathcal{A} u=\Delta u-\langle D \Phi, D u\rangle=e^{\Phi} \operatorname{div}\left(e^{-\Phi} D u\right),
$$

where $\Phi: \mathbb{R}^{N} \rightarrow \mathbb{R}$ is a $C^{2}$ semi-convex function, i.e., there is $\alpha \geq 0$ such that

$$
\Phi_{\alpha}(x):=\Phi(x)+\alpha|x|^{2} / 2 \text { is convex, }
$$

or, equivalently, the matrix $D^{2} \Phi(x)+\alpha I$ is nonnegative definite at each $x$. We emphasize that we do not assume any growth restriction on $\Phi$ or on its derivatives. The natural weight is then $\rho(x)=e^{-\Phi(x)}$ because, as it is easy to check, if $\Omega$ is any open set in $\mathbb{R}^{N}$,

$$
\int_{\Omega} \mathcal{A} u v d \mu=-\int_{\Omega}\langle D u, D v\rangle d \mu, \forall u, v \in C_{0}^{\infty}(\Omega),
$$

if $\mu(d x)=e^{-\Phi(x)} d x$, so that $\mathcal{A}$ is associated to a nice Dirichlet form and it is formally self-adjoint in $L^{2}(\Omega, \mu)$. The aim of this paper is to study the realisation of $\mathcal{A}$ in $L^{2}(\Omega, \mu)$ with Dirichlet boundary condition, i.e., the operator

$$
A: D(A)=\left\{u \in H^{2}(\Omega, \mu) \cap H_{0}^{1}(\Omega, \mu): \mathcal{A} u \in L^{2}(\Omega, \mu)\right\} \rightarrow L^{2}(\Omega, \mu) ; \quad A u=\mathcal{A} u .
$$

Received by the editors April 19, 2004.

2000 Mathematics Subject Classification. Primary 35J70; Secondary 47D07.

Key words and phrases. Elliptic operators, boundary value problems, unbounded coefficients. 
Note that for $u \in H^{2}(\Omega, \mu)$, condition $\mathcal{A} u \in L^{2}(\Omega, \mu)$ is equivalent to requiring $\langle D \Phi, D u\rangle \in L^{2}(\Omega, \mu)$. Our main result is that $A$ is self-adjoint and dissipative, provided $\partial \Omega$ is smooth enough and the normal derivative $\partial \Phi / \partial n$ is bounded from above on $\partial \Omega$. A lot of consequences then follow; see Section 3 .

A natural approach to the study of $\mathcal{A}$ consists in defining an operator $A_{0}$ : $C_{0}^{\infty}(\Omega) \rightarrow L^{2}(\Omega, \mu), A_{0} u=\mathcal{A} u$, in showing that $A_{0}$ is closable, and that its closure is self-adjoint and dissipative. But the problem of the characterisation of the domain of the closure still remains. So, we follow a more direct approach, solving the resolvent equation $\lambda u-A u=f$ for all $\lambda>0$ and $f \in C_{0}^{\infty}(\Omega)$, which is dense in $L^{2}(\Omega, \mu)$. Proving the existence of a solution to $\lambda u-\mathcal{A} u=f$ that vanishes on $\partial \Omega$ is not hard, thanks to the regularity of the data. Estimates of its $H^{1}(\Omega, \mu)$-norm, and uniqueness of the solution in $D(A)$, are easy consequences of the integration formula (5) proved in Lemma 2.2 below. Estimating the second-order derivatives of $u$ is much more delicate, and here the assumptions of semi-convexity and of upper boundedness of $\partial \Phi / \partial n$ are used and play a fundamental role.

This paper is in some sense parallel to the paper [3], where the operator $\mathcal{A}$ was studied in the whole space $\mathbb{R}^{N}$ and in any convex regular open set $\Omega$ with Neumann boundary condition. The conclusions of [3] are similar to the ones of the present paper, but the assumptions on $\Phi$ and $\Omega$ are a bit different, i.e., $\Phi$ is just convex, with no further regularity assumption, and $\Omega$ is convex, too.

\section{The domain of $\mathcal{A}$ with Dirichlet boundary Condition}

Throughout the paper we assume that $\Omega$ is an open set in $\mathbb{R}^{N}$ with sufficiently smooth (at least $C^{2}$ ) boundary. By $L^{2}(\Omega)$ and $H^{k}(\Omega), k \in \mathbb{N}$, we mean the usual $L^{2}$ and Sobolev spaces with respect to the Lebesgue measure. The spaces $H^{k}(\Omega, \mu)$, $k=1,2$, are defined as the set of all $u \in H_{l o c}^{k}(\Omega)$ such that the function $u$ and its partial derivatives up to the order $k$ belong to $L^{2}(\Omega, \mu)$. They are Hilbert spaces with the standard inner products $\langle u, v\rangle=\int_{\Omega}\left(u v+\sum_{|\alpha|=1}^{k} D^{\alpha} u D^{\alpha} v\right) e^{-\Phi} d x$. $H_{0}^{1}(\Omega, \mu)$ is the subspace of $H^{1}(\Omega, \mu)$ consisting of the functions with null trace on the boundary. By $C_{b}^{k}\left(\mathbb{R}^{N}\right)$ we denote the space of bounded functions with bounded derivatives up to order $k$. We say that $\partial \Omega$ is uniformly $C^{k}$ if there exist $r>0$, $m \in \mathbb{N}$ and a (at most countable) family $\left\{B_{j}=B_{r}\left(x_{j}\right), j \in J\right\}$ of balls covering $\partial \Omega$ with at most $m$ overlapping and $C^{k}$-diffeomorphisms $\phi_{j}: B_{j} \rightarrow B_{1}(0)$ such that $\phi_{j}\left(B_{j} \cap \Omega\right)=B_{1}(0) \cap\left\{y_{N}>0\right\}$ and $\sup _{j}\left\|\phi_{j}\right\|_{C^{k}}+\left\|\phi_{j}^{-1}\right\|_{C^{k}}<\infty$.

Lemma 2.1. $C_{0}^{\infty}(\Omega)$ is dense in $L^{2}(\Omega, \mu)$ and in $H_{0}^{1}(\Omega, \mu)$.

Proof. Let $u \in L^{2}(\Omega, \mu)$, or $u \in H_{0}^{1}(\Omega)$. Let $\theta: \mathbb{R}^{N} \rightarrow \mathbb{R}$ be a smooth function such that $0 \leq \theta(x) \leq 1$ for each $x, \theta \equiv 1$ in $B(0,1), \theta \equiv 0$ outside $B(0,2)$, and set $u_{n}(x)=u(x) \theta(x / n)$. Then $u_{n} \rightarrow u$ in $L^{2}(\Omega, \mu)$. Indeed,

$$
\int_{\Omega}\left|u_{n}-u\right|^{2} d \mu \leq \int_{\{x \in \Omega,|x| \geq n\}}|u|^{2} d \mu
$$

which goes to 0 as $n \rightarrow \infty$. If $u \in H_{0}^{1}(\Omega)$, then $u_{n} \rightarrow u$ in $H^{1}(\Omega, \mu)$, because $D u_{n}(x)=\theta(x / n) D u(x)+D \theta(x / n) u(x) / n$. Since each $u_{n}$ has bounded support, it may be approximated in $L^{2}(\Omega)$ (respectively, in $H^{1}(\Omega)$ ) by a sequence of $C_{0}^{\infty}(\Omega)$ functions. Such a sequence also approximates $u_{n}$ in $L^{2}(\Omega, \mu)$ (respectively, in $H^{1}(\Omega, \mu)$ ) because $\mu$ is equivalent to the Lebesgue measure on each compact subset of $\mathbb{R}^{N}$. 
The realisation $A$ of $\mathcal{A}$ in $L^{2}(\Omega, \mu)$ with Dirichlet boundary condition is defined by (B). The following integration formulae will be very useful in what follows.

Lemma 2.2. Let $\psi \in H_{0}^{1}(\Omega, \mu), u \in H^{2}(\Omega, \mu)$ be such that $\mathcal{A} u \in L^{2}(\Omega, \mu)$. Then

$$
\int_{\Omega} \mathcal{A} u \psi d \mu=-\int_{\Omega}\langle D u, D \psi\rangle d \mu .
$$

More generally, if $\psi \in H^{1}(\Omega, \mu)$ and $u \in H^{2}(\Omega, \mu)$ is such that $\mathcal{A} u \in L^{2}(\Omega, \mu)$, then

$$
\int_{\Omega} \mathcal{A} u \psi d \mu=-\int_{\Omega}\langle D u, D \psi\rangle d \mu+\int_{\partial \Omega} \frac{\partial u}{\partial n} \psi e^{-\Phi} d \sigma
$$

where $d \sigma$ denotes the usual Lebesgue surface measure, the last integral is understood as $\lim _{R \rightarrow \infty} \int_{\partial \Omega} \frac{\partial u}{\partial n} \psi \theta(x / R) e^{-\Phi} d \sigma$, and $\theta$ is the function used in Lemma 2.1 .

Proof. The proof of (4) is immediate if $\psi \in C_{0}^{\infty}(\Omega)$, and the statement follows by approximation in the general case. Equality (5) is obtained by approximating $\psi$ by $\psi(x) \theta(x / R)$.

Let us state a consequence of Lemma 2.2

Lemma 2.3. If $\partial \Omega$ is uniformly $C^{2}$ and $u \in H^{2}(\Omega, \mu)$ is such that $\mathcal{A} u \in L^{2}(\Omega, \mu)$, then $\partial u / \partial n$ is in $L^{2}(\partial \Omega, \exp (-\Phi) d \sigma)$. Moreover, there exists $C>0$ such that for every $\varepsilon \in(0,1)$ the following estimate holds:

$$
\int_{\partial \Omega}\left(\frac{\partial u}{\partial n}\right)^{2} e^{-\Phi} d \sigma \leq \varepsilon\left(\|\mathcal{A} u\|_{L^{2}(\Omega, \mu)}^{2}+\left\|\left|D^{2} u\right|\right\|_{L^{2}(\Omega, \mu)}^{2}\right)+\frac{C}{\varepsilon}\||D u|\|_{L^{2}(\Omega, \mu)}^{2} .
$$

Proof. It is sufficient to take $\psi=\langle D u, \mathcal{N}\rangle$ in (5), where $\mathcal{N}$ is any $C_{b}^{1}$ extension to $\mathbb{R}^{N}$ of the normal vector field $n$, and then to use the Hölder inequality.

Lemma 2.2 implies that the operator $A$ is symmetric. In the next theorem we prove that it is self-adjoint if $\Phi$ is smooth enough, and

$$
\frac{\partial \Phi}{\partial n} \leq 0 \text { on } \partial \Omega
$$

Theorem 2.4. Assume that $\partial \Omega \in C^{3}$ and that $\Phi$ satisfies (2) and (6). Then $(A, D(A))$ is self-adjoint and dissipative in $L^{2}(\Omega, \mu)$. Moreover, the map $u \mapsto$ $\left\langle\left(D^{2} \Phi\right) D u, D u\right\rangle$ is continuous from $D(A)$ to $L^{1}(\Omega, \mu)$.

Proof. We have to show that, for $\lambda>0$ and $f \in L^{2}(\Omega, \mu)$, the equation $\lambda u-A u=f$ has a unique solution $u \in D(A)$. Uniqueness is an immediate consequence of Lemma 2.2, taking $\psi=u$ in (5). Concerning existence, we first assume that $f \in C_{0}^{\infty}(\Omega)$ and we show that there is a solution $u \in D(A)$ satisfying

$$
\left\{\begin{array}{l}
\text { (a) }\|u\|_{L^{2}(\Omega, \mu)} \leq \frac{1}{\lambda}\|f\|_{L^{2}(\Omega, \mu)}, \\
\text { (b) }\||D u|\|_{L^{2}(\Omega, \mu)} \leq \frac{1}{\sqrt{\lambda}}\|f\|_{L^{2}(\Omega, \mu)}, \\
\text { (c) }\left\|\left|D^{2} u\right|\right\|_{L^{2}(\Omega, \mu)}+\left\|\left\langle\left(D^{2} \Phi_{\alpha}\right) D u, D u\right\rangle\right\|_{L^{1}(\Omega, \mu)} \leq\left(2+\frac{\alpha}{\lambda}\right)\|f\|_{L^{2}(\Omega, \mu)},
\end{array}\right.
$$

where $\Phi_{\alpha}$ is defined in (2). Using the Lax-Milgram lemma, we find $u \in H_{0}^{1}(\Omega, \mu)$ such that

$$
\lambda \int_{\Omega} u \psi d \mu+\int_{\Omega}\langle D u, D \psi\rangle d \mu=\int_{\Omega} f \psi d \mu, \quad \forall \psi \in H_{0}^{1}(\Omega, \mu) .
$$


By local elliptic regularity, $u \in H_{\mathrm{loc}}^{2}(\Omega)$ and $\lambda u-\mathcal{A} u=f$. In particular, $\mathcal{A} u \in$ $L^{2}(\Omega, \mu)$. Again, by classical elliptic regularity,

$$
u \in C^{2, \beta}(\Omega \cap B(0, R)) \cap H^{3}(\Omega \cap B(0, R))
$$

for every $R>0$ and $\beta<1$.

Now we can prove (17). To prove estimates (a) and (b), we multiply the identity $\lambda u-\mathcal{A} u=f$ by $u$, we integrate over $\Omega$ and we use (4) to get

$$
\int_{\Omega}\left(\lambda u^{2}+|D u|^{2}\right) d \mu=\int_{\Omega} f u d \mu \leq\|f\|_{L^{2}(\Omega, \mu)}\|u\|_{L^{2}(\Omega, \mu)}
$$

which implies that (a) and (b) hold. To prove (c) we differentiate the equation $\lambda u-\mathcal{A} u=f$ with respect to $x_{h}, h+1, \ldots, N$, and we get

$$
\lambda D_{h} u-\Delta\left(D_{h} u\right)+\left\langle D\left(D_{h} \Phi\right), D u\right\rangle+\left\langle D \Phi, D\left(D_{h} u\right)\right\rangle=D_{h} f,
$$

that is,

$$
\lambda D_{h} u-\mathcal{A} D_{h} u+\sum_{k=1}^{N} D_{h k} \Phi D_{k} u=D_{h} f .
$$

Set $\theta_{R}(x)=\theta(x / R)$. Multiplying by $\theta_{R}^{2} D_{h} u$, summing over $h$, and integrating by parts, from (5) we get, since $u \in H^{3}(\Omega \cap B(0, R))$ for every $R$,

$$
\begin{aligned}
& \int_{\Omega}\left\{\theta_{R}^{2}\left(\lambda|D u|^{2}+\left|D^{2} u\right|^{2}+\left\langle D^{2} \Phi D u, D u\right\rangle\right)+2 \sum_{h=1}^{N} \theta_{R}\left\langle D\left(D_{h} u\right), D \theta_{R}\right\rangle D_{h} u\right\} d \mu \\
& =\int_{\partial \Omega} \theta_{R}^{2} \sum_{h=1}^{N} \frac{\partial D_{h} u}{\partial n} D_{h} u e^{-\Phi} d \sigma+\int_{\Omega} \theta_{R}^{2}\langle D f, D u\rangle d \mu
\end{aligned}
$$

Since $f$ has compact support, for $R$ large enough $\theta_{R} \equiv 1$ on the support of $f$. Using (4) again in the last integral, we write it as $-\int_{\Omega} f(\lambda u-f) d \mu$. Thanks to the assumption $D^{2} \Phi \geq-\alpha I$, we obtain

$$
\begin{aligned}
& \int_{\Omega} \theta_{R}^{2}\left(\lambda|D u|^{2}+\left|D^{2} u\right|^{2}+\left\langle\left(D^{2} \Phi_{\alpha}\right) D u, D u\right\rangle\right) d \mu \\
& \leq \int_{\Omega}\left(\alpha \theta_{R}^{2}|D u|^{2}+C R^{-1} \theta_{R}\left|D^{2} u\right||D u|+f(\lambda u-f)\right) d \mu \\
& \quad+\int_{\partial \Omega} \theta_{R}^{2}\left\langle\left(D^{2} u\right) n, D u\right\rangle e^{-\Phi} d \sigma,
\end{aligned}
$$

for a suitable $C>0$, independent of $R$. Using (a) and (b) we get

$$
\int_{\Omega}\left(\alpha \theta_{R}^{2}|D u|^{2}+f(\lambda u-f)\right) d \mu \leq\left(2+\frac{\alpha}{\lambda}\right)\|f\|_{L^{2}(\Omega, \mu)}^{2} .
$$

Moreover,

$$
\int_{\Omega} C R^{-1} \theta_{R}\left|D^{2} u\right||D u| d \mu \leq \frac{C}{2 R} \int_{\Omega} \theta_{R}^{2}\left|D^{2} u\right|^{2} d \mu+\frac{C}{2 R} \int_{\Omega}|D u|^{2} d \mu .
$$

Let us now show that the boundary integral in (99) is negative. Since $u=0$ on $\partial \Omega$, we have $\langle D u, \tau\rangle=0$ and $\left\langle\left(D^{2} u\right) \tau, \tau\right\rangle=0$ for every tangent vector $\tau$ to $\partial \Omega$. Then $D u=(\partial u / \partial n) n$ and $\left\langle\left(D^{2} u\right) n, D u\right\rangle=\left\langle\left(D^{2} u\right) n, n\right\rangle \partial u / \partial n$ at $\partial \Omega$. Therefore 
$\Delta u=\operatorname{trace} D^{2} u=\left\langle\left(D^{2} u\right) n, n\right\rangle$ at $\partial \Omega$, and the equality $\lambda u-\mathcal{A} u=f$ (which is satisfied also at $\partial \Omega$, since $u \in C^{2}(\bar{\Omega} \cap B(0, R))$ for every $R$ ) yields

$$
\Delta u=\frac{\partial \Phi}{\partial n} \frac{\partial u}{\partial n}, \quad\left\langle\left(D^{2} u\right) n, D u\right\rangle=\frac{\partial \Phi}{\partial n}\left(\frac{\partial u}{\partial n}\right)^{2}, \quad \text { at } \partial \Omega
$$

hence

$$
\int_{\partial \Omega} \theta_{R}^{2}\left\langle\left(D^{2} u\right) n, D u\right\rangle e^{-\Phi} d \sigma=\int_{\partial \Omega} \theta_{R}^{2} \frac{\partial \Phi}{\partial n}\left(\frac{\partial u}{\partial n}\right)^{2} e^{-\Phi} d \sigma \leq 0,
$$

thanks to (6). Thus, we have proved that

$$
\int_{\Omega}\left(1-\frac{C}{2 R}\right) \theta_{R}^{2}\left|D^{2} u\right|^{2}+\theta_{R}^{2}\left\langle\left(D^{2} \Phi_{\alpha}\right) D u, D u\right\rangle d \mu \leq\left(2+\frac{\alpha}{\lambda}+\frac{C}{2 R \lambda}\right)\|f\|_{L^{2}(\Omega, \mu)}^{2},
$$

and statement (c) follows by letting $R \rightarrow \infty$.

The general case $f \in L^{2}(\Omega, \mu)$ is easily handled by approximation. Let $\left(f_{n}\right) \subset$ $C_{0}^{\infty}(\Omega)$ be such that $f_{n} \rightarrow f$ in $L^{2}(\Omega, \mu)$ and let $u_{n} \in D(A)$ be such that $\lambda u_{n}-$ $A u_{n}=f_{n}$. The above estimates imply that the sequence $\left(u_{n}\right)$ converges to a function $u$ in $H^{2}(\Omega, \mu)$ and it is readily seen that $u \in D(A), \lambda u-A u=f$ and that (a), (b), and (c) hold.

Condition (6) can be relaxed assuming some more regularity on $\partial \Omega$.

Theorem 2.5. Assume that $\partial \Omega \in C^{3}$ and that it is uniformly $C^{2}$. Let $\Phi$ be a $C^{2}$ function satisfying (2) and

$$
\frac{\partial \Phi}{\partial n} \leq k \text { at } \partial \Omega
$$

for some $k \in \mathbb{R}$. Then $(A, D(A))$ is self-adjoint and dissipative in $L^{2}(\Omega, \mu)$. Moreover, the map $u \mapsto\left\langle\left(D^{2} \Phi\right) D u, D u\right\rangle$ is continuous from $D(A)$ to $L^{1}(\Omega, \mu)$.

Proof. The proof is similar to the proof of Theorem 2.4. For $f \in C_{0}^{\infty}(\Omega), \lambda>0$, let $u \in H_{0}^{1}(\Omega, \mu)$ be the variational solution of the equation $\lambda u-\mathcal{A} u=f$. As in Theorem [2.4 we get estimates (17) (a), (b) and

$$
\begin{aligned}
& \int_{\Omega}\left(1-\frac{C}{2 R}\right) \theta_{R}^{2}\left|D^{2} u\right|^{2} d \mu+\int_{\Omega} \theta_{R}^{2}\left\langle\left(D^{2} \Phi_{\alpha}\right) D u, D u\right\rangle d \mu \\
& \leq\left(2+\frac{\alpha}{\lambda}+\frac{C}{2 R \lambda}\right)\|f\|_{L^{2}(\Omega, \mu)}^{2}+\int_{\partial \Omega} \theta_{R}^{2} \frac{\partial \Phi}{\partial n}\left(\frac{\partial u}{\partial n}\right)^{2} e^{-\Phi} d \sigma .
\end{aligned}
$$

The boundary integral does not exceed

$$
k \int_{\partial \Omega} \theta_{R}^{2}\left(\frac{\partial u}{\partial n}\right)^{2} e^{-\Phi} d \sigma
$$

and it can be estimated as follows (see also Lemma 2.3).

Let us take $\psi=\theta_{R}^{2}\langle D u, \mathcal{N}\rangle$ in (5), where $\mathcal{N}$ is any $C_{b}^{1}$ extension to $\mathbb{R}^{N}$ of the normal vector field $n$, so that, using Hölder inequality, we obtain for every $0<\varepsilon<1$

$$
\int_{\partial \Omega} \theta_{R}^{2}\left(\frac{\partial u}{\partial n}\right)^{2} e^{-\Phi} d \sigma \leq \varepsilon\left(\|\mathcal{A} u\|_{L^{2}(\Omega, \mu)}^{2}+\left\|\theta_{R}\left|D^{2} u\right|\right\|_{L^{2}(\Omega, \mu)}^{2}\right)+\frac{C}{\varepsilon}\|\mid D u\|_{L^{2}(\Omega, \mu)}^{2} .
$$


Since $\mathcal{A} u=\lambda u-f$, writing the last inequality with $\varepsilon k \leq 1 / 2$ and combining it with (11) and with estimates (a), (b), we arrive at

$$
\begin{aligned}
\int_{\Omega}\left(\frac{1}{2}-\frac{C}{2 R}\right) \theta_{R}^{2}\left|D^{2} u\right|^{2} d \mu & +\int_{\Omega} \theta_{R}^{2}\left\langle\left(D^{2} \Phi_{\alpha}\right) D u, D u\right\rangle d \mu \\
& \leq\left(2+\frac{\alpha}{\lambda}+\frac{C}{2 R \lambda}+C_{1}\right)\|f\|_{L^{2}(\Omega, \mu)}^{2}
\end{aligned}
$$

with $C_{1}$ independent of $R$. Letting $R \rightarrow \infty$ we obtain estimate (7) (c) of Theorem 2.4 (with different constants), and from now on the proof follows the same lines as in Theorem 2.4.

Remark 2.6. If $D^{2} \Phi$ is bounded from above, then the mapping $u \mapsto\left\langle\left(D^{2} \Phi\right) D u, D u\right\rangle$ is bounded from $H^{1}(\Omega, \mu)$ to $L^{1}(\Omega, \mu)$ and the last statement of Theorems 2.4 and 2.5 is obvious. But, if $D^{2} \Phi$ is not bounded, the statement is not obvious, and it will be used in the next section to obtain a quantitative Poincaré inequality.

We end this section by showing that $D(A)$ can be strictly contained in $H^{2}(\Omega, \mu) \cap$ $H_{0}^{1}(\Omega, \mu)$.

Example 2.7. We construct a convex function $\phi:[0, \infty) \rightarrow \mathbb{R}$ such that $e^{-\phi}$ and $x^{2} e^{-\phi}$ are in $L^{1}(0, \infty)$ but $\phi^{\prime 2} e^{-\phi} \notin L^{1}(0, \infty)$. Then $u(x)=x$ belongs to $H^{2}(\mu) \cap H_{0}^{1}(\mu)$ but not to $D(A)$. For simplicity, $\phi$ will be nonsmooth. However, smooth versions are easily obtained using straightforward arguments.

Let $0=a_{1}<b_{1}<a_{2}<b_{2}<\cdots$ be points in $[0, \infty)$ such that $b_{j}-a_{j}=1$. Set $1 / l_{j}=a_{j+1}-b_{j}, l_{1}=1$ and define $\phi^{\prime}=1$ in $\left(a_{1}, b_{1}\right), \phi^{\prime}=l_{j}$ in $\left(b_{j}, a_{j+1}\right)$ and $\phi^{\prime}=l_{j-1}$ in $\left(a_{j}, b_{j}\right)$. We have to choose $1=l_{1}<l_{2}<\cdots$ in such a way that $\phi$ satisfies the properties above. First observe that $\phi$ is convex, $\phi^{\prime} \geq 1$, hence $\phi(x) \geq x$ and then $e^{-\phi}, x^{2} e^{-\phi} \in L^{1}(0, \infty)$. Moreover, if $x \in\left(b_{j}, a_{j+1}\right)$, then $\phi(x) \leq j+1+\sum_{i=1}^{j-1} l_{i}$ eand therefore

$$
\int_{b_{j}}^{a_{j+1}} \phi \prime^{2} e^{-\phi} d x \geq l_{j}^{2} \exp \left(-\left(j+1+\sum_{i=1}^{j-1} l_{i}\right)\right) \geq l_{j} \exp \left(-\left(j+1+\sum_{i=1}^{j-1} l_{i}\right)\right) .
$$

Choosing (inductively) $l_{j}=e^{\left(j+1+\sum_{i=1}^{j-1} l_{i}\right)}$ the above integral is bigger than 1 , hence, summing over $j, \phi^{\prime 2}$ does not belong to $L^{1}(\mu)$.

\section{Further properties of $A$}

Under the assumptions of either Theorem 2.4 or Theorem 2.5, since the operator $A$ is self-adjoint and dissipative in $L^{2}(\Omega, \mu)$, it is the infinitesimal generator of an analytic contraction semigroup $T(t)$ in $L^{2}(\Omega, \mu)$. In this section we prove further properties of $T(t)$ and of $A$.

The characterisation of the domain of $(-A)^{1 / 2}$ is a standard consequence of the integration formula (4), as the following proposition shows. Recall that the norm in $H_{0}^{1}(\Omega, \mu)$ is given by $\|u\|_{H_{0}^{1}(\Omega, \mu)}=\|u\|_{L^{2}(\Omega, \mu)}+\|D u\|_{L^{2}(\Omega, \mu)}$.

Proposition 3.1. The domain of $(-A)^{1 / 2}$ is $H_{0}^{1}(\Omega, \mu)$. Therefore, the restriction of $T(t)$ to $H_{0}^{1}(\Omega, \mu)$ is an analytic semigroup in $H_{0}^{1}(\Omega, \mu)$.

Proof. Any $u \in D\left((-A)^{1 / 2}\right)$ is the $L^{2}(\Omega, \mu)$-limit of a sequence of functions $u_{n} \in$ $D(A) \subset H_{0}^{1}(\Omega, \mu)$ which is a Cauchy sequence with respect to the norm $\|u\|_{L^{2}}+$ $\langle-A u, u\rangle_{L^{2}}$. From (4) it follows that $\left(D u_{n}\right)$ is a Cauchy sequence in $L^{2}(\Omega, \mu)$, 
hence $u \in H_{0}^{1}(\Omega, \mu)$. Conversely, let $u \in H_{0}^{1}(\Omega, \mu)$ and let $u_{n} \in C_{0}^{\infty}(\Omega) \subset D(A)$ converge to $u$ in $H^{1}(\Omega, \mu)$. Formula (4) implies that $\left(u_{n}\right)$ is a Cauchy sequence in $D\left((-A)^{1 / 2}\right)$, hence $u \in D\left((-A)^{1 / 2}\right)$.

Corollary 3.2. Under the assumptions of either Theorem 2.4 or Theorem 2.5, $T(t)$ is a symmetric Markov semigroup, that is, a semigroup of self-adjoint positivity preserving operators in $L^{2}(\Omega, \mu)$ that satisfy $\|T(t) f\|_{\infty} \leq\|f\|_{\infty}$ for each $f \in L^{2}(\Omega, \mu) \cap L^{\infty}(\Omega, \mu)$ and $t>0$.

Proof. Since $A$ is self-adjoint, each $T(t)$ is self-adjoint. To prove that each $T(t)$ preserves positivity and that it is a contraction in $L^{\infty}$, we use the Beurling-Deny criteria; see e.g. [4, Theorems 1.3.2, 1.3.3].

As $D\left((-A)^{1 / 2}\right)=H_{0}^{1}(\Omega, \mu)$, then $u \in D\left((-A)^{1 / 2}\right)$ implies $|u| \in D\left((-A)^{1 / 2}\right)$, and

$$
\left\|(-A)^{1 / 2}(|u|)\right\|^{2}=\int_{\Omega}|D(|u|)|^{2} d \mu \leq \int_{\Omega}|D u|^{2} d \mu=\left\|(-A)^{1 / 2} u\right\|^{2},
$$

so that $T(t)$ is positivity-preserving for all $t>0$. Again, since $D\left((-A)^{1 / 2}\right)=$ $H_{0}^{1}(\Omega, \mu)$, if $0 \leq u \in D\left((-A)^{1 / 2}\right)$, then $u \wedge 1 \in D\left((-A)^{1 / 2}\right)$, and

$$
\left\|(-A)^{1 / 2}(u \wedge 1)\right\|^{2}=\int_{\Omega}|D(u \wedge 1)|^{2} d \mu \leq \int_{\Omega}|D u|^{2} d \mu=\left\|(-A)^{1 / 2} u\right\|^{2} .
$$

This implies that $\|T(t) f\|_{\infty} \leq\|f\|_{\infty}$ for each $f \in L^{2}(\Omega, \mu) \cap L^{\infty}(\Omega, \mu)$.

Another immediate consequence of the integration formula (4) is that $A$ is injective: if $u \in D(A)$ and $A u=0$, then $A u \cdot u=0$, and integrating over $\Omega$ we obtain $D u=0$ so that $u$ is constant on each connected component of $\Omega$; since $u$ vanishes at $\partial \Omega$, then $u=0$.

A natural question is now whether 0 is in the resolvent set of $A$. This is true if $D(A)$ is compactly embedded in $L^{2}(\Omega, \mu)$, because in this case the spectrum of $A$ consists of a sequence of isolated eigenvalues. But in general $D(A)$ is not compactly embedded in $L^{2}(\Omega, \mu)$, as the following counterexample shows.

Example 3.3. Let $\varphi: \mathbb{R} \rightarrow \mathbb{R}$ be any convex $C^{2}$ function such that $\varphi(x)=x$ for $x \geq 0$. Set $\Phi(x, y)=\varphi(x)+y^{2}$, and let $\Omega$ be the half-plane $\left\{(x, y) \in \mathbb{R}^{2}: y>0\right\}$. Then $D(A)$ is not compactly embedded in $L^{2}(\Omega, \mu)$.

Proof. Let $\theta \in C_{0}^{\infty}(0, \infty)$ be such that $\int_{0}^{\infty}(\theta(y))^{2} \exp \left(-y^{2}\right) d y=1$, and set for each $n \in \mathbb{N}, n \geq 3$,

$$
u_{n}(x, y)=\frac{x^{n}}{\sqrt{(2 n) !}} \theta(y), \quad x, y \geq 0, \quad u_{n}(x, y)=0 \text { otherwise. }
$$

Since $d \mu=\exp \left(-\varphi(x)-y^{2}\right) d x d y$, then $\left\|u_{n}\right\|_{L^{2}(\Omega, \mu)}=1$ for each $n$. Moreover,

$$
\begin{gathered}
D_{x} u_{n}(x, y)=\frac{n x^{n-1}}{\sqrt{(2 n) !}} \theta(y), \quad D_{y} u_{n}(x, y)=\frac{x^{n}}{\sqrt{(2 n) !}} \theta^{\prime}(y), \quad x>0, \\
D_{x x} u_{n}(x, y)=\frac{n(n-1) x^{n-2}}{\sqrt{(2 n) !}} \theta(y), \quad D_{y y} u_{n}(x, y)=\frac{x^{n}}{\sqrt{(2 n) !}} \theta^{\prime \prime}(y), \quad x>0,
\end{gathered}
$$

and every derivative vanishes for $x \leq 0$. Therefore, $u_{n} \in D(A)$ and $\left\|A u_{n}\right\|_{L^{2}(\Omega, \mu)}$ is bounded by a constant independent of $n$. But no subsequence may converge in 
$L^{2}(\Omega, \mu)$ because

$\left\|u_{n}-u_{m}\right\|_{L^{2}(\Omega, \mu)}^{2}=\int_{0}^{\infty}\left(\frac{x^{2 n}}{(2 n) !}+\frac{x^{2 m}}{(2 m) !}-2 \frac{x^{n+m}}{\sqrt{(2 n) !(2 m) !}}\right) e^{-x} d x=2-2 \frac{(n+m) !}{\sqrt{(2 n) !(2 m) !}}$

and for any fixed $n$ we have

$$
\lim _{m \rightarrow \infty} \frac{(n+m) !}{\sqrt{(2 n) !(2 m) !}}=0, \text { so that } \lim _{m \rightarrow \infty}\left\|u_{n}-u_{m}\right\|_{L^{2}(\Omega, \mu)}^{2}=2 .
$$

In the above example $D_{x} \Phi(x, y)$ is bounded for $x>0$, and the question of whether $D(A)$ is compactly embedded in $L^{2}(\Omega, \mu)$ if $|D \Phi|$ goes to $\infty$ as $|x| \rightarrow \infty$ remains open. In the next proposition we show that the answer is positive if $\Phi$ satisfies an additional (mild) nonoscillation condition.

Proposition 3.4. Assume that $\Phi \in C^{2}\left(\mathbb{R}^{N}\right)$ satisfies $\Delta \Phi \leq a|D \Phi|^{2}+b$ for some $a<1, b \in \mathbb{R}$. Then the map $u \mapsto|D \Phi| u$ is bounded from $H_{0}^{1}(\Omega, \mu)$ to $L^{2}(\Omega, \mu)$. If, in addition, $|D \Phi| \rightarrow \infty$ at infinity, the embedding of $H_{0}^{1}(\Omega, \mu)$ (hence that of $D(A)$ ) in $L^{2}(\Omega, \mu)$ is compact.

Proof. Since $C_{0}^{\infty}(\Omega)$ is dense in $H_{0}^{1}(\Omega, \mu)$ it is sufficient to show that

$$
\||D \Phi| u\|_{L^{2}(\Omega, \mu)} \leq C\|u\|_{H^{1}(\Omega, \mu)}
$$

for some $C>0$ and every $u \in C_{0}^{\infty}(\Omega, \mu)$. Integrating by parts and using Young's inequality we get for every $\varepsilon>0$ and for a suitable $C_{\varepsilon}$

$$
\begin{aligned}
\int_{\Omega}|u|^{2}|D \Phi|^{2} d \mu & =-\int_{\Omega}|u|^{2}\left\langle D \Phi, D e^{-\Phi}\right\rangle d x \\
& =\int_{\Omega}|u|^{2} \Delta \Phi e^{-\Phi} d x+2 \int_{\Omega} u\langle D \Phi, D u\rangle e^{-\Phi} d x \\
& \leq(a+\varepsilon) \int_{\Omega}|u|^{2}|D \Phi|^{2} d \mu+C_{\varepsilon} \int_{\Omega}|D u|^{2} d \mu+b \int_{\Omega}|u|^{2} d \mu .
\end{aligned}
$$

Choosing $\varepsilon$ such that $a+\varepsilon<1$, the first statement follows. Concerning the second one, we observe that for each $\varepsilon>0$ there is $R>0$ such that $|D \Phi| \geq 1 / \varepsilon$ in $\Omega \backslash B(0, R)$. Hence for every $u$ in the unit ball $B$ of $H_{0}^{1}(\Omega)$ we have

$$
\frac{1}{\varepsilon^{2}} \int_{\Omega \backslash B(0, R)}|u|^{2} d \mu \leq \int_{\Omega \backslash B(0, R)}|u|^{2}|D \Phi|^{2} d \mu \leq C^{2} .
$$

Since the embedding of $H^{1}(\Omega \cap B(0, R))$ into $L^{2}(\Omega \cap B(0, R))$ is compact, we can find $\left\{f_{1}, \ldots, f_{k}\right\} \subset L^{2}(\Omega \cap B(0, R))$ such that the balls $B\left(f_{i}, \varepsilon\right) \subset L^{2}\left(\Omega \cap B\left(\tilde{\sigma}_{i}, R\right)\right)$ cover the restrictions of the functions of $B$ to $\Omega \cap B(0, R)$. Denoting by $\tilde{f}_{i}$ the zero-extension of $f_{i}$ to the whole of $\Omega$, it follows that $B \subset \bigcup_{i=1}^{k} B\left(\tilde{f}_{i},(C+1) \varepsilon\right)$, and the proof is complete.

The compactness of the resolvent is a consequence of the logarithmic Sobolev inequality

$$
\int_{\Omega} u^{2} \log (|u|) d \mu \leq \frac{1}{\omega} \int_{\Omega}|D u|^{2} d \mu+\|u\|_{L^{2}(\Omega, \mu)}^{2} \log \left(\|u\|_{L^{2}(\Omega, \mu)}\right),
$$

for all $u \in H_{0}^{1}(\Omega, \mu)$ and some $\omega>0$ (where we set $0 \log 0=0$ ).

In what follows we give sufficient conditions for the validity of (12). 
Proposition 3.5. Let us denote by $\lambda(x)$ the smallest eigenvalue of the matrix $D^{2} \Phi(x)$. Then:

(i) if $\lambda(x) \geq \omega_{0}$ for all $x \in \mathbb{R}^{N}$ then (12) holds with $\omega=\omega_{0}$;

(ii) if $\liminf _{|x| \rightarrow \infty} \lambda(x)>0$, then (12) holds for some $\omega>0$.

Proof. (i) Let $u \in H_{0}^{1}(\Omega, \mu)$ and extend $u$ outside $\Omega$ by setting $u(x)=0$ for $x \notin$ $\Omega$. Then the extension is in $H^{1}\left(\mathbb{R}^{N}, \nu\right)$, where $d \nu(x)=c \exp (-\Phi(x)) d x, c^{-1}=$ $\int_{\mathbb{R}^{N}} \exp (-\Phi) d x \geq 1$. By [3], for each $u \in H^{1}\left(\mathbb{R}^{N}, \nu\right)$ we have

$$
\int_{\mathbb{R}^{N}}|u|^{2} \log |u| d \nu \leq \frac{1}{\omega_{0}} \int_{\mathbb{R}^{N}}|D u|^{2} d \nu+\|u\|_{L^{2}\left(\mathbb{R}^{N}, \nu\right)}^{2} \log \left(\|u\|_{L^{2}\left(\mathbb{R}^{N}, \nu\right)}\right) .
$$

Since $u$ vanishes outside $\Omega$ we easily get

$$
\int_{\Omega}|u|^{2} \log |u| d \mu \leq \frac{1}{\omega_{0}} \int_{\Omega}|D u|^{2} d \mu+\|u\|_{L^{2}(\Omega, \mu)}^{2}\left(\frac{1}{2} \log c+\log \left(\|u\|_{L^{2}(\Omega, \mu)}\right)\right)
$$

and (12) follows since $c \leq 1$.

(ii) The proof is similar to (i), using [11, Theorem 1.3] instead of [3].

Corollary 3.6. Under the assumptions of Proposition 3.5, $H_{0}^{1}(\Omega, \mu)$ is compactly embedded in $L^{2}(\Omega, \mu)$. Therefore, $\sup \sigma(A)<0$. Moreover $T(t)$ maps $L^{2}(\Omega, \mu)$ into $L^{q(t)}(\Omega, \mu)$ with $q(t)=1+e^{\omega t}$, and

$$
\|T(t) f\|_{L^{q(t)}(\Omega, \mu)} \leq\|f\|_{L^{2}(\Omega, \mu)}, \quad t>0, f \in L^{2}(\Omega, \mu) .
$$

Proof. Let $B$ be the unit ball of $H_{0}^{1}(\Omega, \mu)$. Inequality (12) yields the existence of a positive constant $C$ such that $\int_{\Omega}|u|^{2} d \mu \leq C$ for every $u \in B$. Given $t \geq 1$, let $E=\{|u|<t\}$. Then for $R>0$

$$
\begin{aligned}
\int_{\Omega \backslash B(0, R)}|u|^{2} d \mu & \leq \int_{(\Omega \backslash B(0, R)) \cap E} t^{2} d \mu+\frac{1}{\log t} \int_{(\Omega \backslash B(0, R)) \backslash E}|u|^{2} \log |u| d \mu \\
& \leq t^{2} \mu(\Omega \backslash B(0, R))+\frac{C}{\log t}
\end{aligned}
$$

hence, given $\varepsilon>0$, there exists $R>0$ such that $\int_{\Omega \backslash B(0, R)}|u|^{2} d \mu \leq \varepsilon$ for every $u \in B$. As in Proposition 3.4 this proves that $H_{0}^{1}(\Omega, \mu)$ is compactly embedded in $L^{2}(\Omega, \mu)$. The fact that $T(t)$ maps $L^{2}(\Omega, \mu)$ into $L^{q(t)}(\Omega, \mu)$, as well as estimate (13), follow from [5, 6].

A necessary and sufficient condition in order that 0 be in the resolvent of $A$ is that the Poincaré inequality holds, i.e.,

$$
\int_{\Omega}|u|^{2} d \mu \leq \frac{1}{\omega} \int_{\Omega}|D u|^{2} d \mu, \quad u \in H_{0}^{1}(\Omega, \mu)
$$

for some $\omega>0$. More precisely, since $A$ is self-adjoint, then $\langle(-A-\omega I) u, u\rangle \geq 0$ for each $u \in D(A)$ if and only if $\sigma(A+\omega I) \subset(-\infty, 0]$. In other words, (14) holds for each $u \in D(A)$ (or, equivalently, for each $u \in H_{0}^{1}(\Omega, \mu)=D\left((-A)^{1 / 2}\right)$ ) if and only if $\sigma(A) \subset(-\infty,-\omega]$. In this case we have

$$
\|T(t) f\|_{L^{2}(\Omega, \mu)} \leq e^{-\omega t}\|f\|_{L^{2}(\Omega, \mu)}, \quad t>0, f \in L^{2}(\Omega, \mu) .
$$

Indeed, for each $t>0$ and $f \in L^{2}(\Omega, \mu)$,

$$
\frac{d}{d t}\|T(t) f\|^{2}=\int_{\Omega} 2 A T(t) f \cdot T(t) f d \mu=-2\|D T(t) f\|^{2} \leq-2 \omega\|T(t) f\|^{2} .
$$


If $\Omega=\mathbb{R}^{N}$, the Poincaré inequality for functions having zero mean is a consequence of the logarithmic Sobolev inequality (in which case $D(A)$ is compactly embedded in $L^{2}(\Omega, \mu)$ ) and the constant $\omega$ in (14) is the same as in (12); see [10]. This is not true in our setting; see Example 3.9 below. However, in the next proposition we show how to get an explicit estimate of $\omega$ in (14) when (6) holds.

Proposition 3.7. Assume that (6) holds and that there exists $\omega_{0}>0$ such that the map $x \mapsto \Phi(x)-\omega_{0}|x|^{2} / 2$ is convex. Then (14) holds with $\omega=\omega_{0}$.

Proof. We have only to show that $\sigma(A) \subset\left(-\infty,-\omega_{0}\right]$. Corollary 3.6 yields that the resolvent of $A$ is compact, hence $\sigma(A)$ consists of eigenvalues. If $\lambda u-A u=0$ for some $\lambda \in \mathbb{R}$ and $0 \neq u \in D(A)$, we write (8) with $f=0$ and let $R \rightarrow \infty$. Since the boundary integral is nonpositive and $D^{2} \Phi \geq \omega_{0} I$ we get $\left(\lambda+\omega_{0}\right) \int_{\Omega}|D u|^{2} \leq 0$. Since $u$ is not a constant, then $D u \neq 0$ and $\lambda \leq-\omega_{0}$. This concludes the proof.

Let us again consider Example 3.3 and show that, in general, the Poincaré inequality does imply that the embedding $D(A) \subset L^{2}(\Omega, \mu)$ is compact.

Example 3.8. We use the same notation as in Example 3.3 Proposition 3.7 applied to the one-dimensional function $y \mapsto y^{2}, y>0$, yields

$$
\int_{0}^{\infty}|u(x, y)|^{2} e^{-y^{2}} d y \leq \frac{1}{2} \int_{0}^{\infty}\left|D_{y} u(x, y)\right|^{2} e^{-y^{2}} d y, \quad \text { a.e. } x \in \mathbb{R}, u \in H_{0}^{1}(\Omega, \mu) .
$$

Multiplying by $e^{-\phi(x)}$ and integrating with respect to $x \in \mathbb{R}$, we deduce

$$
\int_{\Omega}|u(x, y)|^{2} d \mu \leq \frac{1}{2} \int_{\Omega}\left|D_{y} u(x, y)\right|^{2} d \mu
$$

so that the Poincaré inequality holds, even if $D(A)$ is not compactly embedded in $L^{2}(\Omega, \mu)$, as we have shown in Example 3.3 .

If assumption (6) is replaced by the boundedness of $\partial \Phi / \partial n$ at $\partial \Omega$ and still $\Phi(x)-\omega^{2}|x|^{2}$ is convex, the constant $\omega$ in (14) may also depend on the constant $k$ in (10), as we show in the following example.

Example 3.9. Let $N=1$ and let $\mathcal{A} u=u^{\prime \prime}-x u^{\prime}$ be the Ornstein-Uhlenbeck operator. Here $\Phi(x)=x^{2} / 2$, hence $D^{2} \Phi \equiv 1$ and (12) holds with $\omega=1$. Let $\Omega_{a}=(-\infty, a)$ and set $u(x)=a-x$. Then $u \in D(A)$ and

$$
\int_{-\infty}^{a}\left|u^{\prime}\right|^{2} d \mu\left(\int_{-\infty}^{a}|u|^{2} d \mu\right)^{-1} \rightarrow 0
$$

as $a \rightarrow \infty$. This shows that the spectrum of $A$ in $L^{2}\left(\Omega_{a}, \mu\right)$ is not contained in $(-\infty,-1]$ for large $a$, hence the constant $\omega$ in (14) is smaller than 1 .

\section{REFERENCES}

[1] M. Bertoldi, S. Fornaro: Gradient estimates in parabolic problems with unbounded coefficients, Studia Math. 165 (2004), 221-254. MR2109509

[2] S. CERrAI: Second order PDE's in finite and infinite dimensions. A probabilistic approach, Lecture Notes in Mathematics 1762, Springer-Verlag, Berlin (2001). MR1840644 (2002j:35327)

[3] G. Da Prato, A. Lunardi: Elliptic operators with unbounded drift coefficients and Neumann boundary condition, J. Diff. Eqns. 198 (2004), 35-52. MR2037749 (2004k:35066)

[4] E. B. Davies: Heat Kernels and Spectral Theory, Cambridge U. P. (1989). MR0990239 (90e:35123) 
[5] L. Gross: Logarithmic Sobolev Inequalities, Amer. J. Math. 97 (1975), 1061-1083. MR 0420249 (54:8263)

[6] L. Gross: Logarithmic Sobolev Inequalities and Contractivity Properties of Semigroups, in: Dirichlet Forms (G. Dell'Antonio and U. Mosco Eds.), Lecture Notes in Mathematics 1563, Springer-Verlag, Berlin (1993), 54-88. MR1292277|(95h:47061)

[7] G. Metafune, D. Pallara, V. Vespri: $L^{p}$-estimates for a class of elliptic operators with unbounded coefficients in $\mathbb{R}^{N}$, Houston J. Math. 31 (2005), 605-620.

[8] G. Metafune, A. Rhandi, J. Prüss, R. Schnaubelt: $L^{p}$ regularity for elliptic operators with unbounded coefficients, preprint.

[9] P.RABIER: Elliptic problems on $\mathbb{R}^{N}$ with unbounded coefficients in classical Sobolev spaces, Math. Z. 249 (2005), 1-30. MR.2106968

[10] O.S. Rothaus: Logarithmic Sobolev Inequalities and the Spectrum of Schrödinger operators, J. Funct. Anal. 42 (1981), 110-120. MR0620582 (83f:58080b)

[11] Feng-Yu Wang: Logarithmic Sobolev inequalities: conditions and counterexamples, J. Operator Theory 46 (2001), 183-197. MF1862186 (2003g:58060)

Dipartimento di Matematica, Università di Parma, Parco Area delle Scienze 53, 43100 PARMA, ITALY

E-mail address: lunardi@unipr.it

Dipartimento di Matematica "Ennio De Giorgi", Università di Lecce, C.P.193, 73100, LECCE, ITALY

E-mail address: giorgio.metafune@unile.it

Dipartimento di Matematica "Ennio De Giorgi", Università di Lecce, C.P.193, 73100, LECCE, ITALY

E-mail address: diego.pallara@unile.it 